\title{
High-throughput sequence analysis reveals variation in the relative abundance of components of the bacterial and fungal microbiota in the rhizosphere of Ginkgo biloba
}

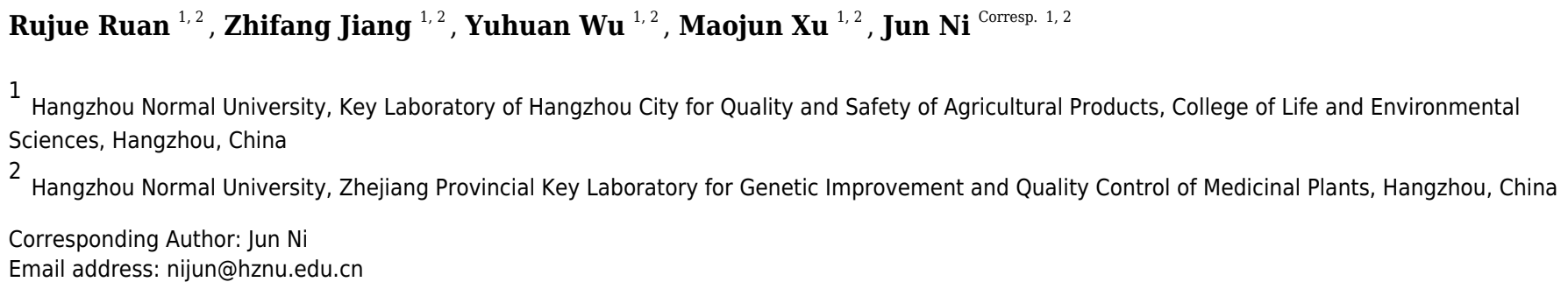

Background. The narrow region of soil, in contact with and directly influenced by plant roots, is called the rhizosphere. Microbes living in the rhizosphere are considered to be important factors for the normal growth and development of plants. In this research, the structural and functional diversities of microbiota between the Ginkgo biloba root rhizosphere and the corresponding bulk soil were investigated.

Methods. Three independent replicate sites were selected, and triplicate soil samples were collected from the rhizosphere and the bulk soil at each sampling site. The communities of bacteria and fungi were investigated using high-throughput sequencing of the 16S rRNA gene and the internal transcribed spacer (ITS) of the rRNA gene, respectively.

Results. A number of bacterial genera showed significantly different abundance in the rhizosphere compared to the bulk soil, including Bradyrhizobium, Rhizobium, Sphingomonas, Streptomyces and Nitrospira. Functional enrichment analysis of bacterial microbiota revealed consistently increased abundance of ATP-binding cassette (ABC) transporters and decreased abundance of two-component systems in the rhizosphere community, compared to the bulk soil community. In contrast, the situation was more complex and inconsistent for fungi, indicating the independency of the rhizosphere fungal community on the local microenvironment. 
1 High-throughput sequence analysis reveals variation

2 in the relative abundance of components of the

3 bacterial and fungal microbiota in the rhizosphere of

4 Ginkgo biloba

5 Rujue Ruan ${ }^{1,2}$, Zhifang Jiang ${ }^{1,2}$, Yuhuan $\mathrm{Wu}^{1,2}$, Maojun $\mathrm{Xu}^{1,2}, \mathrm{Jun}^{\mathrm{Ni}}{ }^{1,2 *}$

6

7

\section{8}

${ }^{1}$ Key Laboratory of Hangzhou City for Quality and Safety of Agricultural Products, College of Life and Environmental Sciences, Hangzhou Normal University, Hangzhou 310018, China.

${ }^{2}$ Zhejiang Provincial Key Laboratory for Genetic Improvement and Quality Control of Medicinal Plants, Hangzhou Normal University, Hangzhou 310018, China.

\section{Corresponding Author:}

Jun Ni

NO. 16 Xuelin Street, Hangzhou, 310018, China.

Email address: nijun@hznu.edu.cn

\section{Abstract}

Background. The narrow region of soil, in contact with and directly influenced by plant roots, is called the rhizosphere. Microbes living in the rhizosphere are considered to be important factors for the normal growth and development of plants. In this research, the structural and functional diversities of microbiota between the Ginkgo biloba root rhizosphere and the corresponding bulk soil were investigated.

Methods. Three independent replicate sites were selected, and triplicate soil samples were collected from the rhizosphere and the bulk soil at each sampling site. The communities of bacteria and fungi were investigated using high-throughput sequencing of the 16S rRNA gene and the internal transcribed spacer (ITS) of the rRNA gene, respectively.

Results. A number of bacterial genera showed significantly different abundance in the rhizosphere compared to the bulk soil, including Bradyrhizobium, Rhizobium, Sphingomonas, Streptomyces and Nitrospira. Functional enrichment analysis of bacterial microbiota revealed consistently increased abundance of ATP-binding cassette (ABC) transporters and decreased abundance of two-component systems in the rhizosphere community, compared to the bulk soil community. In contrast, the situation was more complex and inconsistent for fungi, indicating the independency of the rhizosphere fungal community on the local microenvironment.

\section{Introduction}

Microorganisms act not only as pathogens, causing plant diseases (Chisholm et al. 2006), but also as neighbors or symbionts, playing important roles in plant productivity and health by providing a plethora of functional activities. Plant-associated microorganisms are often referred 
to as the plant's second genome and have received substantial attention in recent years (Berg et al. 2014; Turner et al. 2013). For studying the microbial diversity of prokaryotes, such as bacteria, PCR amplification of the ubiquitous 16S ribosomal RNA (rRNA) gene, coupled with high-throughput sequencing technologies, have allowed identification of even rare nonculturable microbial species in a sample (Caporaso et al. 2011; Youssef et al. 2009). For studying eukaryotic microbes, such as fungi, the hypervariable internal transcribed spacer (ITS) rDNA sequences are often used to assess taxonomic diversity (Bachy et al. 2013; Bengtsson - Palme et al. 2013).

The narrow region of soil, in contact with and directly influenced by plant roots, is called the rhizosphere. The rhizosphere is critical to normal plant growth and development, with all the inorganic and organic substances exchanged between the root and soil occurring through this zone. On the other hand, plants also modify the rhizosphere to better adapt to the ever-changing environment (Ryan et al. 2001). Significant differences have been reported with respect to the physical, chemical and biological characteristics of the plant rhizosphere soil compared to the surrounding bulk soil, and this phenomenon is known as the "rhizosphere effect" (Hartmann et al. 2008). Specifically, enormous numbers of microbes live in the rhizosphere, forming a complex plant-associated microbial community, and this community is considered to be crucial for plant health (Berendsen et al. 2012). Ginkgo biloba, a "living fossil", is an important long-lived native Chinese tree species with no living relatives (Zhou 2009). It is used as a medical plant, with ginkgo herb being commonly used as an herbal dietary supplement and for the treatment of many ailments, including Alzheimer's disease (Rimbach et al. 2001). It is also one of the horticultural tree species which is now widely planted in China. Moreover, G. biloba is regarded as a valuable municipal tree in many cities in China (Figure S1, Table S1). Due to its importance to human health and the environment, much research has been carried out on ginkgo, especially focused on the flavonoid biosynthesis pathway in ginkgo leaves (Ni et al. 2018a; Ni et al. 2018b; Ni et al. 2017). However, our knowledge of the relationship between ginkgo and the microbiota in its rhizosphere is still limited.

In this research, the $16 \mathrm{~S}$ and ITS sequences were analyzed in bacteria and fungi, respectively, to estimate the diversity of microbiota in the ginkgo root rhizosphere compared to the bulk soil. A number of bacterial genera were found to accumulate in the rhizosphere, while there were also some bacterial genera for which the abundance decreased in rhizosphere. In contrast, the situation with the abundance of fungal genera in the rhizosphere versus the bulk soil was complex and inconsistent across the three sampling sites, indicating that fungal abundance was relatively independent of the rhizosphere microenvironment. Our results identified a number of bacterial genera, the abundance of which differed between the rhizosphere and the bulk soil, indicating a complex relationship between ginkgo and soil microbes.

\section{Materials \& Methods}

\section{Sampling and DNA extraction}


79 Three independent replicate sites in the campus of Hangzhou Normal University were selected

80 for soil sample collection in August 2017. Ginkgo roots were collected at a depth of about $20 \mathrm{~cm}$

81 below ground level. Large soil aggregates were removed by shaking the roots, and the

82 rhizosphere soil was defined as the remaining soil particles adhering to the roots. The bulk soil

83 was collected about $10 \mathrm{~m}$ away from the ginkgo trees at a depth of about $20 \mathrm{~cm}$ below ground

84 level. The bulk soil was also free from roots of other plants. For the collection of rhizosphere soil,

85 roots were transferred to a $15 \mathrm{ml}$-Falcon tube containing $2.5 \mathrm{ml}$ phosphate-buffered saline (PBS)

86 solution, and were sonicated 30 times (Scientz-JY92-IIN, China), each consisting of $30 \mathrm{~s}$ pulses

87 at $160 \mathrm{~W}$, with breaks between pulses of $30 \mathrm{~s}$. After removing the roots, the suspension was

88 centrifuged at $1,500 \mathrm{x}$ g for $20 \mathrm{~min}$, and the pelleted soil was used for DNA extraction. The

89 PowerSoil $^{\mathrm{TM}}$ DNA Isolation Kit (MoBio, USA) was used to extract the DNA from the

90 rhizosphere and bulk soil samples, following the manufacturer's instructions.

91

\section{PCR amplification and sequencing of amplicon libraries}

The DNA samples were individually amplified by PCR using primers S-D-Bact-0341-b-S-17 (5'-CCTACGGGNGGCWGCAG-3'), S-D-Bact-0785-a-A-21 (5'-

GACTACHVGGGTATCTAATCC-3') for 16S rDNA in bacteria (Klindworth et al. 2012), and ITS1 (5'-TCCGTAGGTGAACCTGCGG-3'), ITS2 (5'-GCTGCGTTCTTCATCGATGC-3') for ITS in fungi (Sreenivasaprasad et al. 1996). Each $30 \mu$ PCR reaction mixture contained 5 10 ng DNA template, $15 \mu 12 \times$ Master Mix (Phusion ${ }^{\circledR}$ High-Fidelity PCR Master Mix with GC Buffer, New England Biolabs, USA), with each primer in the reaction mixture being supplied at a concentration of $3 \mu \mathrm{M}$. Cycling conditions included initial denaturation at $98^{\circ} \mathrm{C}$ for $1 \mathrm{~min}$, followed by 30 cycles of denaturation at $98^{\circ} \mathrm{C}$, each cycle lasting $10 \mathrm{~s}$, annealing at $50{ }^{\circ} \mathrm{C}$ for 30 $\mathrm{s}$, and extension at $72{ }^{\circ} \mathrm{C}$ for $30 \mathrm{~s}$; a final extension phase was performed at $72{ }^{\circ} \mathrm{C}$ for $5 \mathrm{~min}$. The PCR products were separated on a $2 \%(\mathrm{w} / \mathrm{v})$ agarose gel. The DNA bands between $400 \mathrm{bp}$ and $450 \mathrm{bp}$ were collected. The DNA was extracted from the gel slices using the GeneJET gel extraction kit (Thermo Scientific, USA). The amplicon libraries were constructed using NEB Next ${ }^{\circledR}$ Ultra $^{\mathrm{TM}}$ DNA Library Prep Kit for Illumina (New England Biolabs, USA) according to the manufacturer's protocol. The qualified libraries were sequenced on an Illumina Hiseq2500 platform and $250 \mathrm{bp}$ paired-end reads were generated. The raw sequencing data have been deposited in NCBI Sequence Read Archive (SRA) under accession number PRJNA565829 for bacteria (https://www.ncbi.nlm.nih.gov/sra/PRJNA565829), and PRJNA566252 for fungi (https://www.ncbi.nlm.nih.gov/sra/PRJNA566252).

\section{Sequencing data analysis, OTU production and annotation}

The original paired-end reads, cutting off the barcode and primer sequences, were merged to total tags with FLASH (v.1.2.11) (Magoc \& Salzberg 2011). The total tags were filtered by Qiime (v.1.9.1) (Bokulich et al. 2013; Caporaso et al. 2010). These tags were then compared with the reference database (Gold database, http://drive5.com/uchime/uchime_download.html) using UCHIME algorithms (http://www.drive5.com/usearch/manual/uchime_algo.html) to detect 
119

120

121

122

123

124

125

126

127

128

129

130

131

132

133

134

135

136

137

138

139

140

141

142

143

144

145

146

147

148

149

150

151

152

153

154

155

156

157

158

and remove the chimera sequences (Edgar et al. 2011; Haas et al. 2011). After that, these tags were termed effective tags and were ready for further analysis.

The effective tags with $\geq 97 \%$ similarity were assigned to the same operational taxonomic units (OTUs) using Uparse (v8.1.1861), and the sequence with the highest frequency of occurrence in each OTU was selected as the representative sequence for further annotation (Edgar 2013). For each representative sequence, the annotation was performed using the uclust method and the Silva database to the level of kingdom, phylum, class, order, family, genus and species, to determine the community composition of each sample. OTU abundance information was normalized using a standard of sequence number corresponding to the sample with the least sequences (For bacteria, the number was 22755. For fungi, the number was 34819). Subsequent analyses were all performed based on these normalized data.

\section{Alpha and beta diversity analysis}

Alpha diversity was applied to analyze complexity of species diversity for a community through four indices, including abundance-based coverage (ACE) index, Shannon diversity index, phylogenetic diversity (PD)_whole_tree and Good's coverage index. All these indices from our samples were calculated with QIIME (Version 1.9.1) and displayed using R software (Version 3.2.2). The ACE estimator (http://www.mothur.org/wiki/Ace) was selected to determine community richness, while the Shannon index (http://www.mothur.org/wiki/Shannon) was used to estimate community diversity, and Good's coverage index (http://www.mothur.org/wiki/Coverage) was used to quantify sequencing depth.

For beta diversity analysis, principal component analysis (PCA) was applied to reduce the dimension of the original variables using the FactoMineR package and ggplot2 package in $\mathrm{R}$ software (Version 3.2.2). Unweighted Pair-Group Method with Arithmetic Means (UPGMA) Clustering was performed as a type of hierarchical clustering method to interpret the distance matrix using average linkage, and was conducted using QIIME software (Version 1.9.1).

\section{Correlation analysis and KEGG functional analysis}

Correlation analysis (using Spearman's rank correlation analysis) was performed based on the changes in species abundances in different communities, using CCREPE (http://huttenhower.sph.harvard.edu/ccrepe). The first 100 groups were displayed by Cytoscape (http://chianti.ucsd.edu/cytoscape-3.2.1/). To analyze the function of the microbiota, Tax4Fun (http://tax4fun.gobics.de) was used to determine the Kyoto Encyclopedia of Genes and Genomes (KEGG) estimate. The variation analysis was carried out based on the functional abundance of the samples. 


\section{Results}

160 Quality metrics of microbiome sequencing data from the rhizosphere and bulk soil samples

161

162

163

164

165

166

167

168

169

170

171

172

173

174

175

176

177

178

179

180

181

182

183

184

185

186

187

188

189

190

191

192

193

194

195

196

197

198

To investigate the differences in microbiota between the rhizosphere of ginkgo roots and the bulk soil, 16S rDNA amplicon sequencing for bacteria and ITS rDNA sequencing for fungi were performed on DNA samples extracted from the ginkgo rhizosphere and from bulk soil collected $10 \mathrm{~m}$ from the nearest ginkgo tree. Three independent replicate sites were chosen for sample collections. Within each site, triplicate samples were collected from both the rhizosphere and the bulk soil. For bacterial $16 \mathrm{~S}$ rDNA analysis, more than 40,000 total tags were obtained in each sample except for S-2 (38,717 total tags) in site 1, and R-4 (34,905 total tags) in site 2. After trimming and filtering, more than $80 \%$ of the tags were assigned to be effective tags that were ready for further analysis (Table 1). For fungal ITS rDNA analysis, more than 40,000 total tags were obtained in each sample except for S-8 (36,779 total tags) in site 3. Similarly, more than $85 \%$ of the tags were assigned to be effective tags that were ready for further analysis. In addition, more than $98 \%$ base calls were $99 \%$ confidence (Q20) and more than $94 \%$ base calls were $99.9 \%$ confidence (Q30) for each sample with respect to both bacteria and fungi. These results indicated that the sequencing data from $16 \mathrm{~S}$ and ITS were acceptable (Table 1).

\section{OTU analysis and annotation of microbiome sequences}

To study the species diversities of the microbiome from the sequencing data, the annotated clean reads (taxon reads) were clustered into OTUs. An average ( \pm standard deviation) number of $2823.0 \pm 283.7$ OTUs was obtained for bacteria, with a corresponding number of $430.8 \pm 146.2$ OTUs for fungi (Table 2). These taxon tags were also classified to different levels of taxonomy, and three technical replicates from the same soil and site exhibited similar taxonomic patterns, which showed that the repeatability of the experiment was acceptable. Different taxonomic patterns were observed between samples from rhizosphere and bulk soil from each of the three collection sites. This indicated marked differences in the microbiota between the rhizosphere and the bulk soil (Figure 1, Table S2 and Table S3). We also investigated the ten most-abundant items with respect to different taxonomic levels of phylum, class, order, family and genus. For bacteria, the extent of variation increased from phylum to genus (Figure S2 to Figure S6). Specifically, the frequencies of the ten most abundant genera in rhizosphere soil were quite different in the bulk soil (Figure S6). In contrast, we found very different patterns at almost every level of taxonomy in the fungi, which indicated even greater variation in the fungal microbiota than in the bacterial microbiota when comparing communities in the rhizosphere with those in the bulk soil (Figure S2 to Figure S6).

\section{Alpha and beta diversity analysis of samples}

To quantify alpha diversity, the diversities of bacteria and fungi in each sample were analyzed based on the diversity indices of ACE, Shannon, PD_whole_tree and Good's_coverage. The Good's_coverage index for all the samples was greater than 0.95 for bacteria (Table S4) and greater than 0.99 for fungi (Table S5), demonstrating that the sequencing depth was acceptable. 
199 For bacteria, generally, the three indices of ACE, Shannon and PD_whole tree were lower in the
200 rhizosphere soil compared to that in the bulk soil. Specifically, all three indices were

200

201

202

203

204

205

206

207

208

209

210

211

212

213

214

215

216

217

218

219

220

221

222

223

224

225

226

227

228

229

230

231

232

233

234

235

236

237

238 significantly different in site 1, with similar variation tendencies in sites 2 and 3 (Figure 2). This demonstrated the selective effect on bacterial diversity of the rhizosphere. In contrast, the differences in these indices between rhizosphere and bulk soil were different or even opposite in different sites for the fungi. For example, all three indices decreased significantly in the rhizosphere soil from site 1, but increased significantly in the rhizosphere soil of site 3 compared to that in the corresponding bulk soil (Figure 2). These results indicated that the effects of the ginkgo rhizosphere on the distribution of the bacteria may be different from that of the fungi.

For beta diversity, PCA analysis was carried out to cluster bacterial and fungal communities in rhizosphere and bulk soil, according to the different sampling sites. At the OTU level, PC1 explained $19.33 \%$ and PC2 $11.59 \%$ of the total variation in bacteria, and the rhizosphere samples were clearly distinguishable from the bulk soil samples (Figure 3A). In contrast, PC1 explained $13.71 \%$ and PC2 $10.05 \%$ of the total variation in fungi; samples from the rhizosphere and bulk soil did not separate clearly with respect to the fungi (Figure S7A). We also performed UPGMA cluster analysis and built cluster trees for the samples. Using this approach, all the samples collected from the rhizosphere were distinguishable from the samples from the bulk soil with respect to bacteria, while the situation was less clear-cut with respect to the fungi (Figure 3B, Figure S7B).

\section{Variations in the microbiota in the ginkgo rhizosphere at the genus level}

To take a closer look at the variation in the microbiota of the ginkgo rhizosphere, the 35 most abundant genera of bacteria were compared between the rhizosphere and the bulk soil. Interestingly, the abundance of most of the genera varied between the rhizosphere and the bulk soil (Figure 4). Several genera showed increased abundance in the rhizosphere, including Bradyrhizobium, Rhizobium, Sphingomonas and Streptomyces. In contrast, there were also some genera which showed decreased abundance in the rhizosphere compared to that in the bulk soil, including Nitrospira (Figure 4). We also examined the relative abundance values in the rhizosphere and bulk soil of the 35 highest-frequency fungal genera, and found that, although some genera showed significantly different abundances between rhizosphere and bulk soil at one collection site, this was not consistent across the other sites (Figure S8). This indicated that, for individual fungal genera, the selective effect (positive or negative) of the rhizosphere was not significant as it was in bacteria.

\section{Correlation and KEGG functional analysis in bacteria}

Based on the changes in abundance of different genera in different soil samples, the relationships between the abundance values of the various bacterial genera were determined. It was obvious that Rhizobium and Pantoea dominated the microbiota. Rhizobium was the most abundant genus, and had a significant positive relationship with Bosea. Pantoea was also highly abundant and had very close relationships with many other genera, including Bradyrhizobium (Figure S9). The

Peer) reviewing PDF | (2019:06:38264:2:0:NEW 8 Oct 2019) 
239 relationship network of these genera indicated a complex functional collaboration within the 240 microbiota. To analyze the functional diversities of bacteria, the KEGG functional enrichment 241 analysis of bacterial microbiota was compared between the rhizosphere and the bulk soil. The 242 frequency of ATP-binding cassette (ABC) transporters was enriched significantly in the 243 rhizosphere while the frequency of the two-component system decreased significantly in the 244 rhizosphere (Figure S10). This indicated a functional divergence of bacterial microbiota in 245 response to the rhizosphere of ginkgo roots.

246

\section{Discussion}

248 With the development of the ginkgo-based pharmaceutical industry and of ginkgo horticulture, it

249

250

251

252

253

254

255

256

257

258

259

260

261

262

263

264

265

266

267

268

269

270

271

272

273

274

275

276

277

278 is increasingly important to fully understand the different aspects of ginkgo biology, including the relationship with its root microbiota. Most of the previous research on ginkgo had focused on the biosynthesis pathways of various bioactive compounds present in ginkgo leaves, which are raw materials for the pharmaceutical industry. On the other hand, little research has been carried out on ginkgo roots. To our knowledge, this research represents the first report on the relationship between ginkgo roots and the soil microbiota.

In this study, the $16 \mathrm{~S}$ rDNA in bacteria and the ITS rDNA sequences in fungi were amplified and sequenced. We did not find the contaminant sequences from chloroplast, mitochondrial or nuclear DNA, which have frequently occurred in related research (Beckers et al. 2016). This finding showed that our PCR approach was optimized and suitable for this research. Considering the complex nature of the soil environment, which may cause changes in microbiota composition, we chose three different and independent sites for sample collection. Only those changes which occurred in all three sites were discussed.

One of the important findings in this study was the accumulation in the rhizosphere of species of Rhizobium and its fellow rhizobial genus Bradyrhizobium. Rhizobium and Bradyrhizobium are nitrogen-fixing bacteria which induce the development of nodules in the roots of legume plant hosts (Long 1996). In addition, flavonoids are widely accepted to be regulators of symbiotic interactions, acting as specific signals between plant hosts and Rhizobium (Mierziak et al. 2014). Considering that flavonoids are one of the most important groups of secondary plant metabolites in ginkgo (Kleijnen \& Knipschild 1992), it is possible that ginkgo root cells secrete flavonoids, which act as specific signals to attract the accumulation of Rhizobium and Bradyrhizobium in the rhizosphere. However, despite the accumulation of Rhizobium and Bradyrhizobium in the rhizosphere, we did not find any nodules on ginkgo roots. This might be due to the lack of Nod factor receptors or to defects of the subsequent kinase cascade in ginkgo, which is crucial to nodule formation in legumes (Gage 2004; Smit et al. 2007). Following the recent publication of the draft genome of G. biloba (Guan et al. 2016), it would be interesting to identify the missing steps in ginkgo which are associated with its inability to form nodules.

We also observed an accumulation of Sphingomonas in the ginkgo rhizosphere. Bacteria within the genus Sphingomonas share the common capacity to degrade a broad range of aromatic compounds (Fredrickson et al. 1995). Thus, the accumulation of Sphingomonas in the 
279 rhizosphere indirectly suggested the secretion of different aromatic secondary metabolites from 280 ginkgo roots, which attracted the accumulation of aromatics-consuming Sphingomonas species. 281 Streptomyces is the largest genus of the Actinobacteria, with more than 500 species having 282 been described (Euzeby 2008). Streptomyces not only produces a volatile metabolite, geosmin, 283 which result in the distinct "earthy" odor of soil, but also produces antibiotics, which they use to

284

285

286

287

288

289

290

291

292

293

294

295

296

297

298

299

300

301

302

303

304

305

306

307

308

309

310

311

312

313

314

315

316

317

compete with other bacteria for resources. A number of them have been developed as antifungals, antibiotics and chemotherapeutic drugs to improve human health (Raja \& Prabakarana 2011). The benefits to ginkgo of Streptomyces accumulation in the rhizosphere are currently unknown, but this phenomenon could increase the complexity of the composition of the microbiota in the rhizosphere.

In contrast to those bacterial genera which accumulated in the ginkgo rhizosphere, there were also some genera which decreased in the rhizosphere compared to the bulk soil. The genus Nitrospira consists of a group of species which are widely distributed in many natural environments (Bartosch et al. 2002), and they are considered to play important roles in the nitrogen cycle in both water and soil (Hayatsu et al. 2008). Despite the potential advantage of an exogenous nitrate supply to ginkgo, Nitrospira did not accumulate in the rhizosphere but rather was present at lower frequencies in the rhizosphere than in the bulk soil. This decline may due to the inhibitory effects of antibiotics produced by other rhizobacteria (Streptomyces, for instance), which accumulated in the rhizosphere. Alternatively, the decline may be caused by the complex secondary metabolites secreted by ginkgo roots. It has been reported that flavonoids, secreted by root cells, had both positive and negative effects on nodule formation by nitrogen-fixing bacteria (Cooper 2004; Khandual 2007). Thus, it is possible that certain compounds secreted by ginkgo root cells prevented the accumulation of certain bacteria, including Nitrospira.

$\mathrm{ABC}$ transporters are integral membrane proteins that couple the transport of substrates across lipid bilayers to the hydrolysis of ATP (Hollenstein et al. 2007). In bacteria, ABC transporters are important factors catalyzing the uptake of nutrients and the efflux of toxic or antimicrobial agents, which are crucial for bacterial survival (Davidson \& Chen 2004). The accumulation in the rhizosphere of bacteria enriched with respect to $\mathrm{ABC}$ transporters is consistent with the function of the rhizosphere, which mediates the exchange of inorganic and organic substances between the roots and the soil (Ryan et al. 2001). Two-component systems are characterized by a sensor kinase consisting of a signal-recognition domain with unique specificity, coupled to an auto-kinase domain, and they are the major means by which bacteria recognize and respond to a range of environmental stimuli (Hoch 2000). For the bacteria in the rhizosphere, the frequency of two-component systems decreased compared to that in the bulk soil. We propose that bacteria must cope with different environmental stimuli in the soil. For the bacteria in the rhizosphere, the microenvironment is greatly affected by the plants. These bacteria are partially "protected" by the rhizosphere, and there is no need for these bacteria to employ numerous two-component systems to cope with the different challenges from the ever- changing environment as would be the case for the bacteria in the bulk soil.

Peerj reviewing PDF | (2019:06:38264:2:0:NEW 8 Oct 2019) 
318

319

320

321

322

323

324

325

326

327

328

329

330

331

332

333

334

335

336

337

338

339

340

341

342

343

344

345

346

347

348

349

350

351

352

353

354

355

Compared with the clear changes in bacterial frequency between the rhizosphere and bulk soil, the responses of fungal frequency between the two soil types varied between the different collection sites. Many fungal genera accumulated in the rhizosphere of one collection site, yet decreased at other collection sites (Figure S8). Considering the different and complex subcellular structures of fungi, it is possible that the substances secreted by root cells and bacteria have relatively little effect on the distribution of the fungal microbiota.

\section{Conclusions}

In this research, the structural and functional diversities of microbiota between the Ginkgo biloba root rhizosphere and the corresponding bulk soil were investigated. A number of bacterial genera showed significantly different abundance in the rhizosphere compared to the bulk soil, including Bradyrhizobium, Rhizobium, Sphingomonas, Streptomyces and Nitrospira. Functional enrichment analysis of bacterial microbiota revealed consistently increased abundance of ATPbinding cassette $(\mathrm{ABC})$ transporters and decreased abundance of two-component systems in the rhizosphere community, compared to the bulk soil community. In contrast, the situation was more complex and inconsistent for fungi, indicating the independency of the rhizosphere fungal community on the local microenvironment. This study was the first attempt to characterize the microbiota in the ginkgo rhizosphere, which indicated a complex relationship between ginkgo and the microbial communities in the soil.

\section{Acknowledgements}

We thank Prof. Huizhong Wang from Hangzhou Normal University for suggestions and constructive criticism of this manuscript.

\section{References}

Bachy C, Dolan JR, Lopez-Garcia P, Deschamps P, and Moreira D. 2013. Accuracy of protist diversity assessments: morphology compared with cloning and direct pyrosequencing of 18S rRNA genes and ITS regions using the conspicuous tintinnid ciliates as a case study. ISME J 7:244-255. 10.1038/ismej.2012.106

Bartosch S, Hartwig C, Spieck E, and Bock E. 2002. Immunological detection of Nitrospira-like bacteria in various soils. Microbial Ecology 43:26-33.

Beckers B, Op De Beeck M, Thijs S, Truyens S, Weyens N, Boerjan W, and Vangronsveld J. 2016. Performance of 16s rDNA Primer Pairs in the Study of Rhizosphere and Endosphere Bacterial Microbiomes in Metabarcoding Studies. Front Microbiol 7:650. 10.3389/fmicb.2016.00650

Bengtsson - Palme J, Ryberg M, Hartmann M, Branco S, Wang Z, Godhe A, Wit P, Sánchez - García M, Ebersberger I, and Sousa F. 2013. Improved software detection and extraction of ITS1 and ITS2 from ribosomal ITS sequences of fungi and other eukaryotes 
356

357

358

359

360

361

362

363

364

365

366

367

368

369

370

371

372

373

374

375

376

377

378

379

380

381

382

383

384

385

386

387

388

389

390

391

392

393

394

395

for analysis of environmental sequencing data. Methods in ecology and evolution 4:914919.

Berendsen RL, Pieterse CMJ, and Bakker PAHM. 2012. The rhizosphere microbiome and plant health. Trends in Plant Science 17:478-486. 10.1016/j.tplants.2012.04.001

Berg G, Grube M, Schloter M, and Smalla K. 2014. Unraveling the plant microbiome: looking back and future perspectives. Front Microbiol 5:148. 10.3389/fmicb.2014.00148

Bokulich NA, Subramanian S, Faith JJ, Gevers D, Gordon JI, Knight R, Mills DA, and Caporaso JG. 2013. Quality-filtering vastly improves diversity estimates from Illumina amplicon sequencing. Nat Methods 10:57-59. 10.1038/nmeth.2276

Caporaso JG, Kuczynski J, Stombaugh J, Bittinger K, Bushman FD, Costello EK, Fierer N, Pena AG, Goodrich JK, Gordon JI, Huttley GA, Kelley ST, Knights D, Koenig JE, Ley RE, Lozupone CA, McDonald D, Muegge BD, Pirrung M, Reeder J, Sevinsky JR, Turnbaugh PJ, Walters WA, Widmann J, Yatsunenko T, Zaneveld J, and Knight R. 2010. QIIME allows analysis of high-throughput community sequencing data. Nat Methods 7:335-336. 10.1038/nmeth.f.303

Caporaso JG, Lauber CL, Walters WA, Berg-Lyons D, Lozupone CA, Turnbaugh PJ, Fierer N, and Knight R. 2011. Global patterns of 16S rRNA diversity at a depth of millions of sequences per sample. Proceedings of the National Academy of Sciences 108:4516-4522.

Chisholm ST, Coaker G, Day B, and Staskawicz BJ. 2006. Host-Microbe Interactions: Shaping the Evolution of the Plant Immune Response. Cell 124:803-814. 10.1016/j.cell.2006.02.008

Cooper JE. 2004. Multiple responses of rhizobia to flavonoids during legume root infection. Advances in Botanical Research: Elsevier, 1-62.

Davidson AL, and Chen J. 2004. ATP-binding cassette transporters in bacteria. Annual review of biochemistry 73:241-268.

Edgar RC. 2013. UPARSE: highly accurate OTU sequences from microbial amplicon reads. Nat Methods 10:996-998. 10.1038/nmeth.2604

Edgar RC, Haas BJ, Clemente JC, Quince C, and Knight R. 2011. UCHIME improves sensitivity and speed of chimera detection. Bioinformatics 27:2194-2200.

10.1093/bioinformatics/btr381

Euzeby J. 2008. Genus streptomyces. List of Prokaryotic names with Standing in Nomenclature. Fredrickson J, Balkwill D, Drake G, Romine M, Ringelberg D, and White D. 1995. Aromaticdegrading Sphingomonas isolates from the deep subsurface. Applied and Environmental Microbiology 61:1917-1922.

Gage DJ. 2004. Infection and invasion of roots by symbiotic, nitrogen-fixing rhizobia during nodulation of temperate legumes. Microbiology and Molecular Biology Reviews 68:280300 .

Guan R, Zhao Y, Zhang H, Fan G, Liu X, Zhou W, Shi C, Wang J, Liu W, Liang X, Fu Y, Ma K, Zhao L, Zhang F, Lu Z, Lee SM, Xu X, Yang H, Fu C, Ge S, and Chen W. 2016. Draft genome of the living fossil Ginkgo biloba. Gigascience 5:49. 10.1186/s13742-016-0154-1 
396

397

398

399

400

401

402

403

404

405

406

407

408

409

410

411

412

413

414

415

416

417

418

419

420

421

422

423

424

425

426

427

428

429

430

431

432

433

434

435

Haas BJ, Gevers D, Earl AM, Feldgarden M, Ward DV, Giannoukos G, Ciulla D, Tabbaa D, Highlander SK, Sodergren E, Methe B, DeSantis TZ, Petrosino JF, Knight R, and Birren BW. 2011. Chimeric 16S rRNA sequence formation and detection in Sanger and 454pyrosequenced PCR amplicons. Genome Res 21:494-504. 10.1101/gr.112730.110

Hartmann A, Rothballer M, and Schmid M. 2008. Lorenz Hiltner, a pioneer in rhizosphere microbial ecology and soil bacteriology research. Plant and Soil 312:7-14. $10.1007 / \mathrm{s} 11104-007-9514-\mathrm{z}$

Hayatsu M, Tago K, and Saito M. 2008. Various players in the nitrogen cycle: Diversity and functions of the microorganisms involved in nitrification and denitrification. Soil Science \& Plant Nutrition 54:33-45. 10.1111/j.1747-0765.2007.00195.x

Hoch JA. 2000. Two-component and phosphorelay signal transduction. Current opinion in microbiology 3:165-170.

Hollenstein K, Dawson RJP, and Locher KP. 2007. Structure and mechanism of ABC transporter proteins. Current Opinion in Structural Biology 17:412-418. 10.1016/j.sbi.2007.07.003

Khandual S. 2007. Flavonoids as Signaling Molecules and Regulators of Root Nodule Development. Dynamic Soil, Dynamic Plant 1:83-94.

Kleijnen J, and Knipschild P. 1992. Ginkgo biloba. The Lancet 340:1136-1139. 10.1016/01406736(92)93158-J

Klindworth A, Pruesse E, Schweer T, Peplies J, Quast C, Horn M, Glöckner FO. 2012. Evaluation of general 16S ribosomal RNA gene PCR primers for classical and nextgeneration sequencing-based diversity studies. Nucleic Acids Research 41: e1-e1. Long SR. 1996. Rhizobium symbiosis: nod factors in perspective. The Plant Cell 8:1885-1898. Magoc T, and Salzberg SL. 2011. FLASH: fast length adjustment of short reads to improve genome assemblies. Bioinformatics 27:2957-2963. 10.1093/bioinformatics/btr507

Mierziak J, Kostyn K, and Kulma A. 2014. Flavonoids as Important Molecules of Plant Interactions with the Environment. Molecules 19:16240.

Ni J, Dong L, Jiang Z, Yang X, Chen Z, Wu Y, and Xu M. 2018a. Comprehensive transcriptome analysis and flavonoid profiling of Ginkgo leaves reveals flavonoid content alterations in day-night cycles. PLoS One 13:e0193897. 10.1371/journal.pone.0193897

Ni J, Dong L, Jiang Z, Yang X, Sun Z, Li J, Wu Y, and Xu M. 2018b. Salicylic acid-induced flavonoid accumulation in Ginkgo biloba leaves is dependent on red and far-red light. Industrial Crops and Products 118:102-110. https://doi.org/10.1016/j.indcrop.2018.03.044

Ni J, Hao J, Jiang Z, Zhan X, Dong L, Yang X, Sun Z, Xu W, Wang Z, and Xu M. 2017. NaCl induces flavonoid biosynthesis through a putative novel pathway in post-harvest Ginkgo leaves. Frontiers in Plant Science 8:920. 10.3389/fpls.2017.00920

Raja A, and Prabakarana P. 2011. Actinomycetes and drug-an overview. American Journal of Drug Discovery and Development 1:75-84.

Rimbach G, Gohil K, Matsugo S, Moini H, Saliou C, Virgili F, Weber SU, and Packer L. 2001. Induction of glutathione synthesis in human keratinocytes by Ginkgo biloba extract (EGb761). Biofactors 15:39-52. 
436 Ryan P, Delhaize E, and Jones D. 2001. Function and Mechanism of Organic Anion Exudation 437 from Plant Roots. Annu Rev Plant Physiol Plant Mol Biol 52:527-560.

$438 \quad$ 10.1146/annurev.arplant.52.1.527

439 Smit P, Limpens E, Geurts R, Fedorova E, Dolgikh E, Gough C, and Bisseling T. 2007.

440 Medicago LYK3, an entry receptor in rhizobial nodulation factor signaling. Plant

$441 \quad$ Physiology 145:183-191.

442 Sreenivasaprasad S, Sharada K, Brown AE, Mills PR. 1996. PCR-based detection of

443 Colletotrichum acutatum on strawberry. Plant Pathology 45: 650-655.

444 Turner TR, James EK, and Poole PS. 2013. The plant microbiome. Genome Biol 14:209.

$445 \quad$ 10.1186/gb-2013-14-6-209

446 Youssef N, Sheik CS, Krumholz LR, Najar FZ, Roe BA, and Elshahed MS. 2009. Comparison

447 of species richness estimates obtained using nearly complete fragments and simulated

448 pyrosequencing-generated fragments in 16S rRNA gene-based environmental surveys.

$449 \quad$ Applied and Environmental Microbiology 75:5227-5236.

450 Zhou Z-Y. 2009. An overview of fossil Ginkgoales. Palaeoworld 18:1-22.

$451 \quad$ 10.1016/j.palwor.2009.01.001 
Figure 1

Different levels of taxonomic distribution of taxon tags.

Site 1 , Site 2 and Site 3 are the three sample collection sites. R-1 to R-9 are samples collected from rhizosphere. S-1 to S-9 are samples collected from the bulk soil. Different colors represent different levels of taxonomy. (A) Bacteria. (B) Fungi. K, kingdom. P, phylum. C, class. O, order. F, family. G, genus. S, species. 

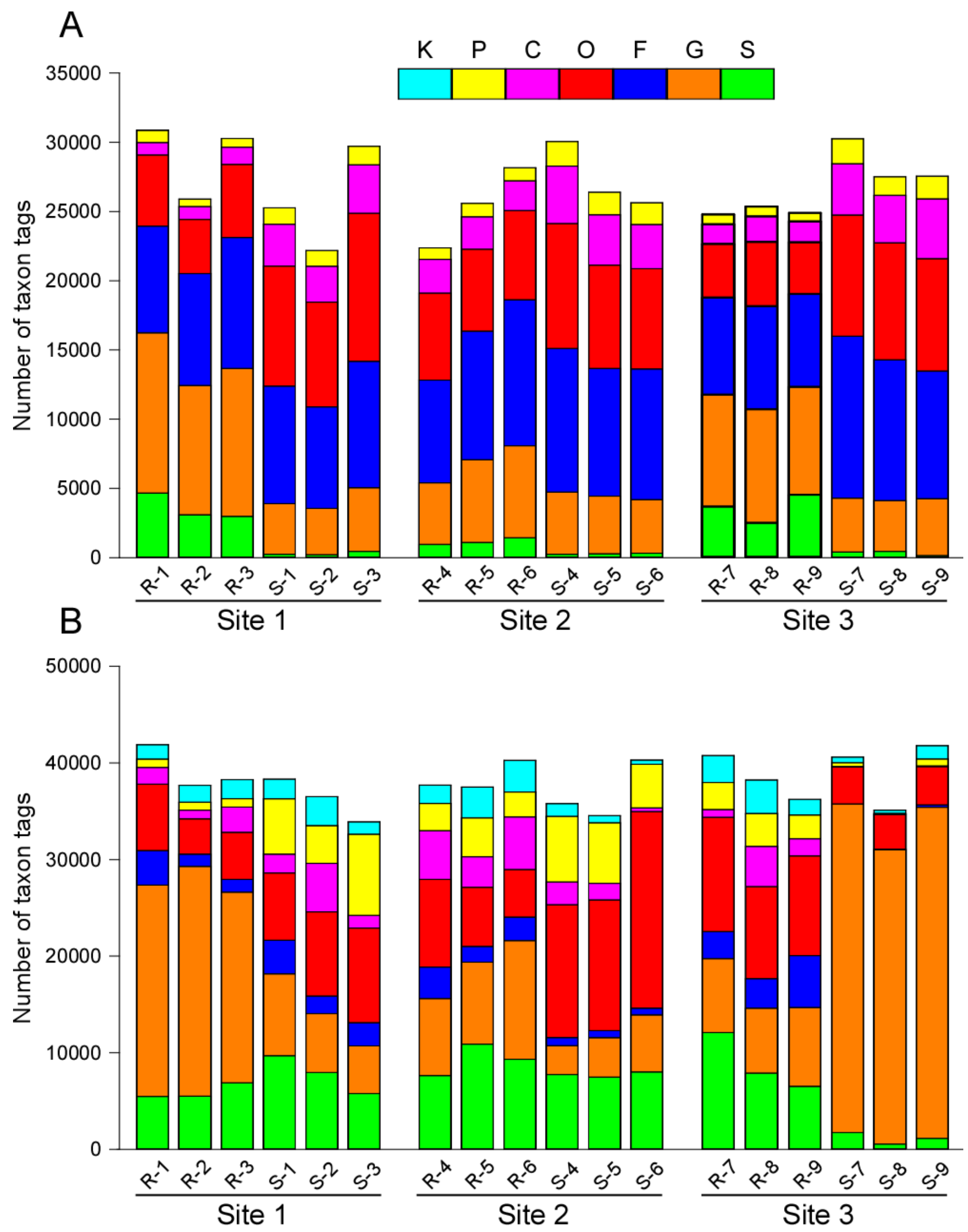
Figure 2

Alpha diversity of the bacterial and fungal communities.

(A), (C) and (E), three indices for bacteria. (B), (D) and (F), three indices for fungi. Alpha diversity estimates represent three biological replicates for the rhizosphere $(R)$ and the bulk soil (S) from the three sites. Significant changes $(P<0.05)$, determined by the Tukey test, are marked by an asterisk. NS, not significant. 
A

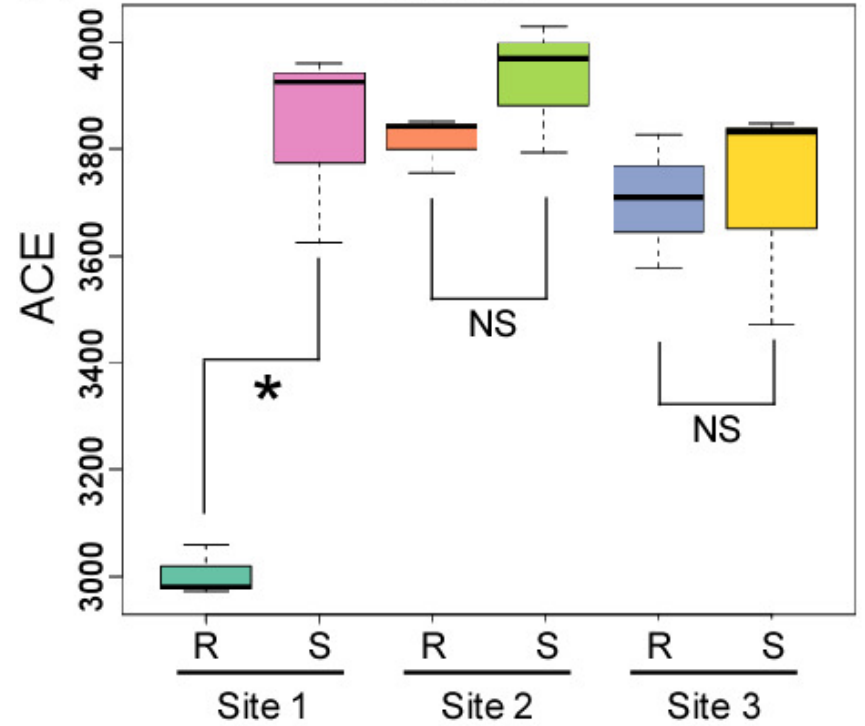

C

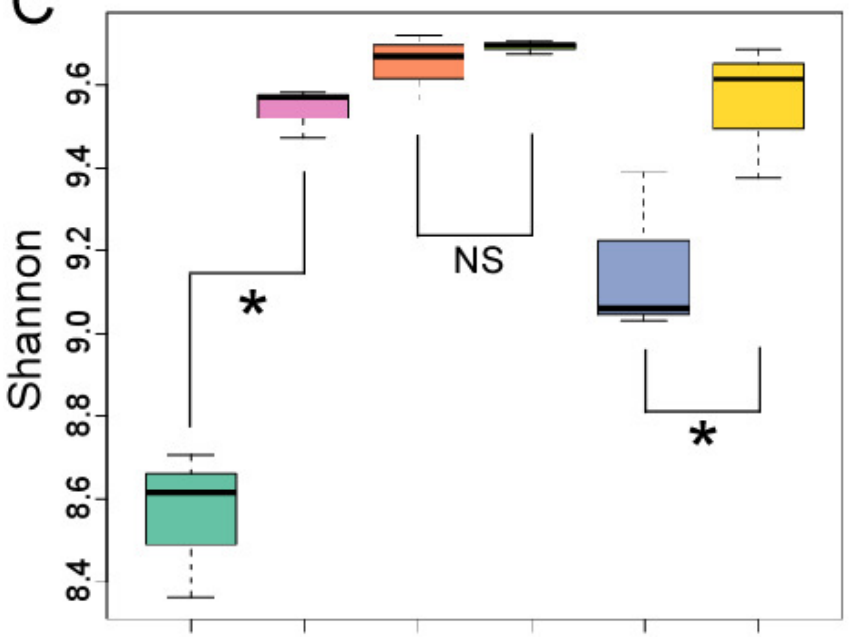

E

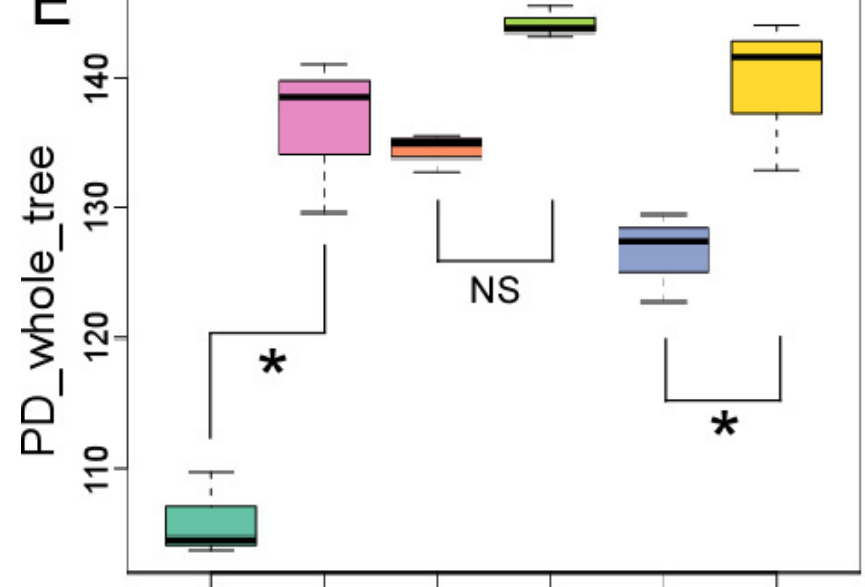

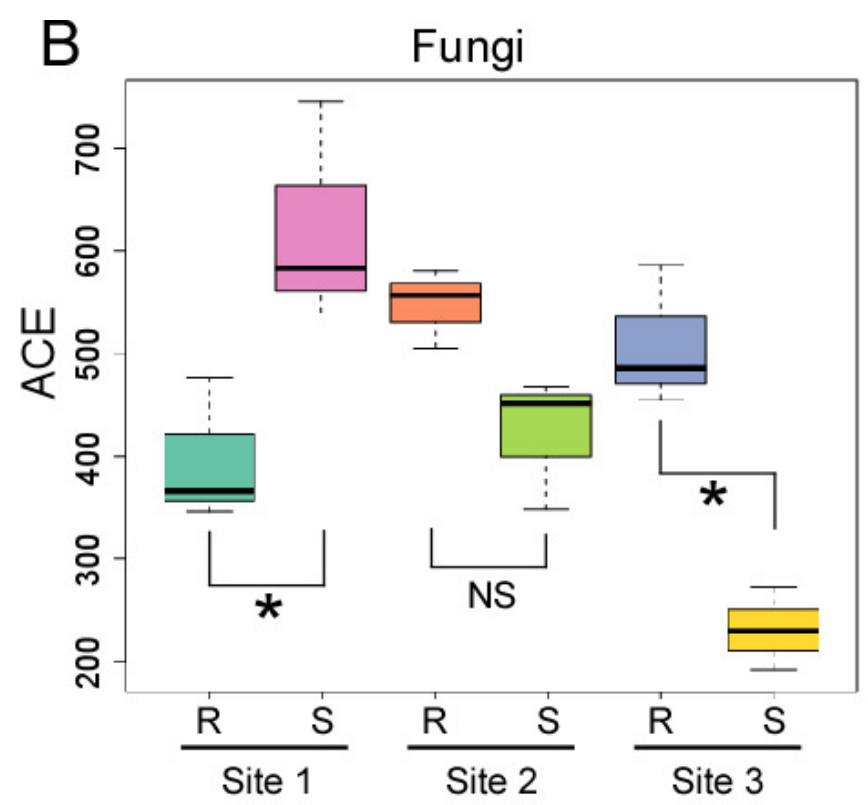
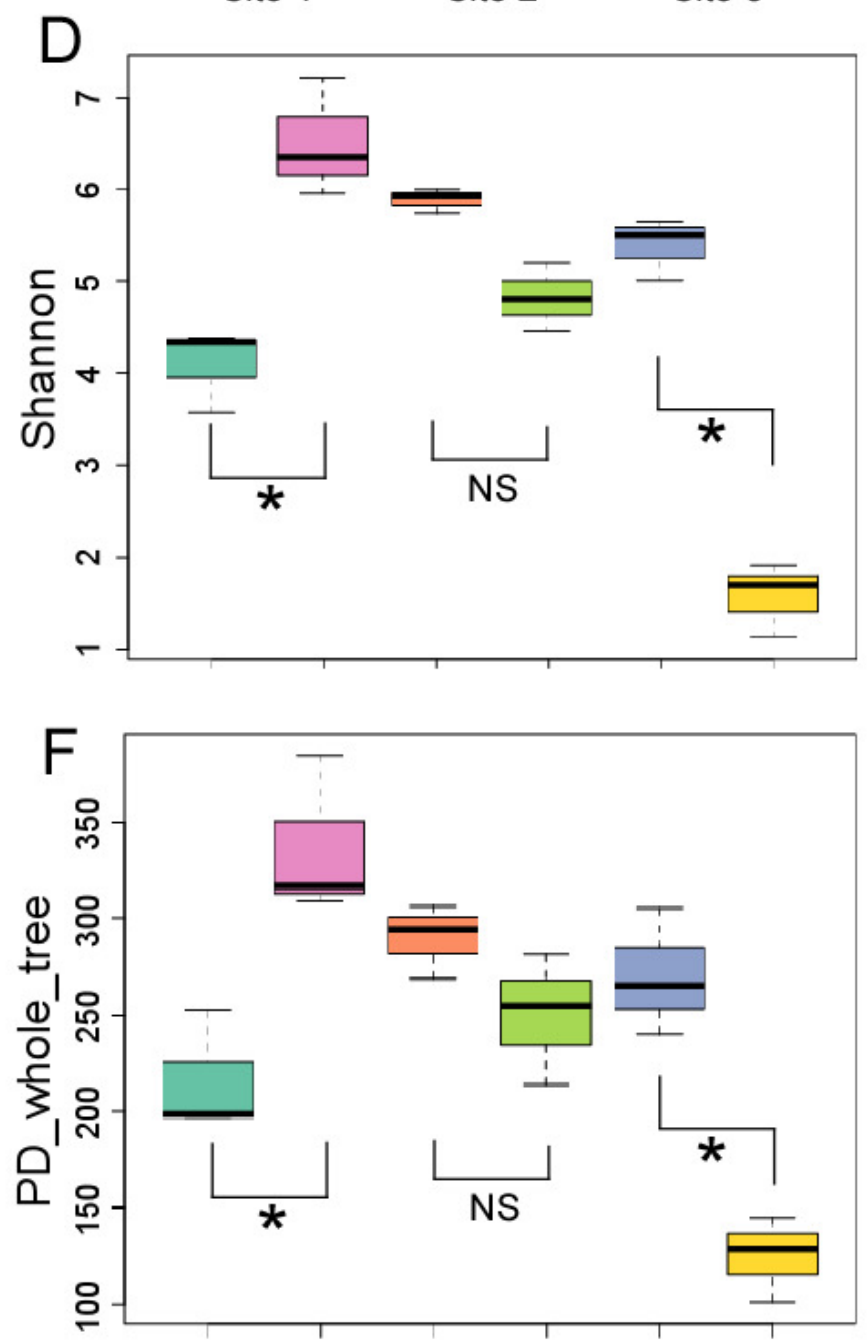
Figure 3

Beta diversity of the bacterial communities.

(A) PCA analysis. Samples from rhizosphere and control soil are marked. (B) UPGMA cluster analysis. Samples collected from rhizosphere are marked in blue, and samples collected from the bulk soil are marked in red. 

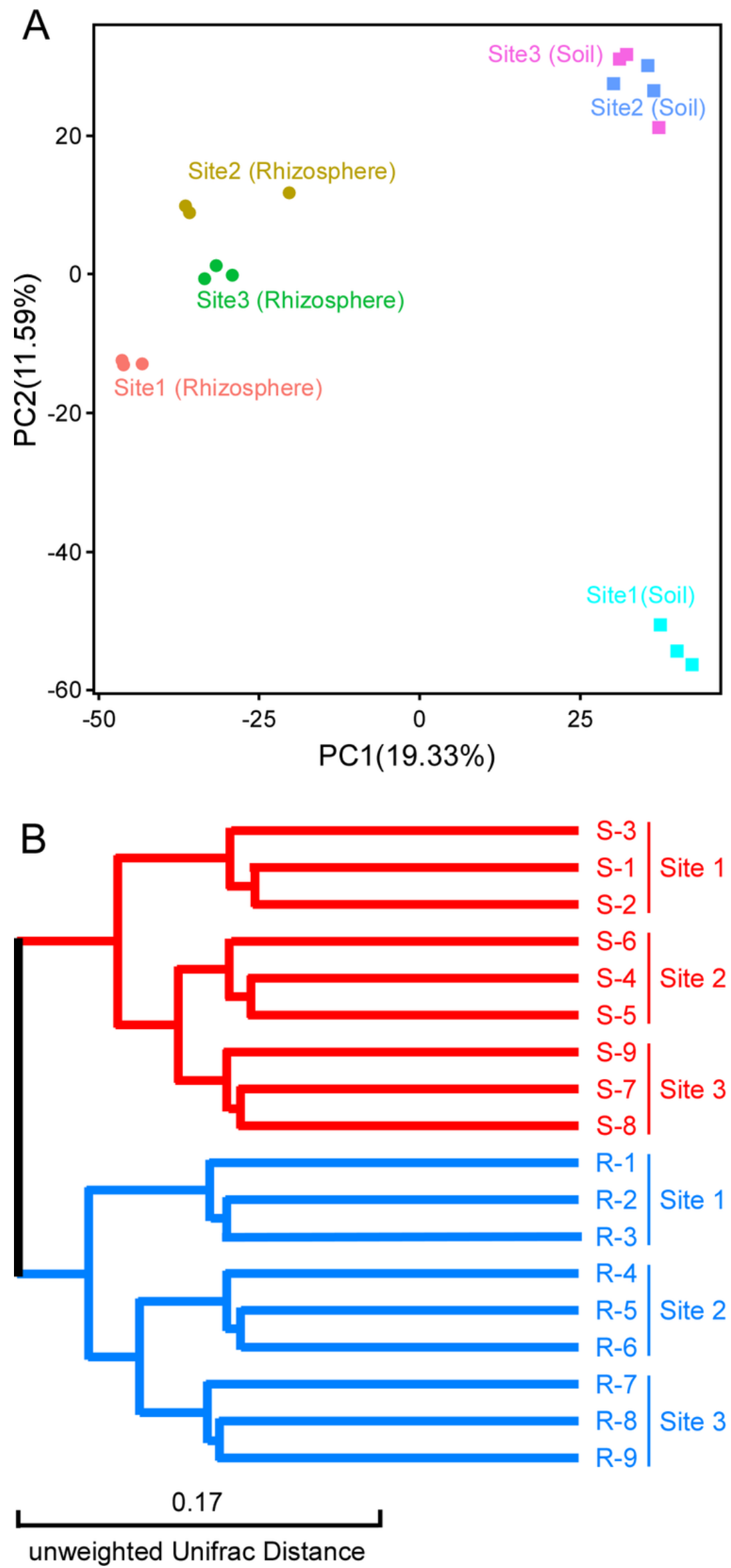


\section{Figure 4}

The abundance of the 35 most-abundant genera of bacteria in the rhizosphere and the bulk soil from the three sites.

The taxonomies of genera to the level of phylum are marked by different colors. The relative abundances of the 35 genera are scaled by a Z-score color gradient bar. The red colored data represent genera that have higher abundance than average. The blue colored data represent genera that have lower abundance than average. A Z-score of 0 represents a genus abundance value that is equal to the average abundance value. 


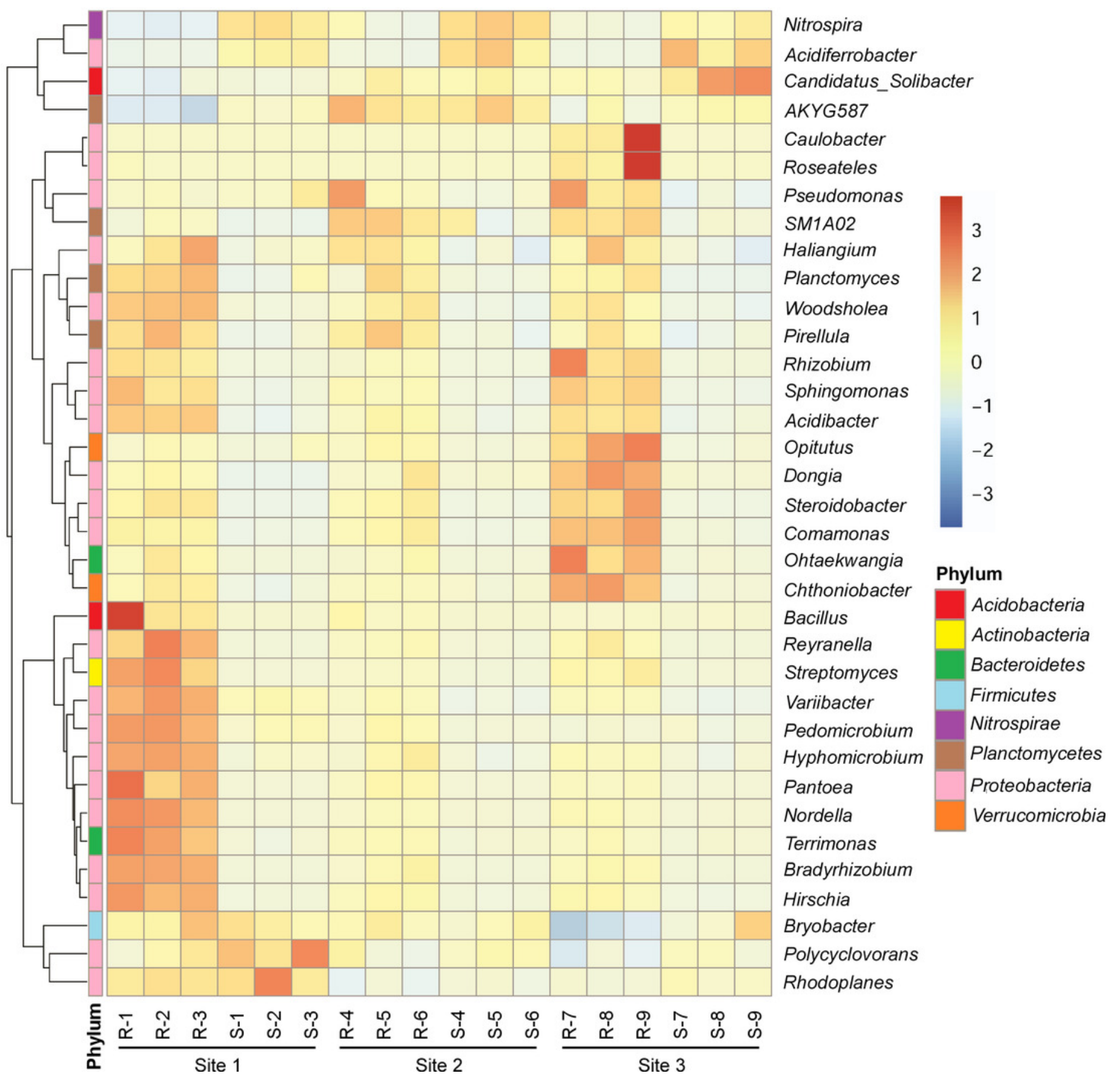




\section{Table $\mathbf{1}$ (on next page)}

The raw sequencing data and quality control of $16 \mathrm{~S}$ and ITS.

R-1 to R-9 are samples collected from the rhizosphere. S-1 to S-9 are samples collected from the bulk soil. AvgLen = average lengths of the clean reads. Q20 and Q30 are the ratio of bases with quality value more than 20 and 30 (error rates of less than $1 \%$ and $0.1 \%$, respectively) in the clean reads. 


\begin{tabular}{|c|c|c|c|c|c|c|c|c|c|c|c|c|c|}
\hline \multirow{2}{*}{\multicolumn{2}{|c|}{$\begin{array}{l}18 \text { samples } \\
\text { from } 3 \text { sites }\end{array}$}} & \multicolumn{6}{|c|}{$16 S$} & \multicolumn{6}{|c|}{ ITS } \\
\hline & & \multirow{2}{*}{$\begin{array}{c}\begin{array}{c}\text { Total } \\
\text { reads }\end{array} \\
44,196\end{array}$} & \multirow{2}{*}{$\begin{array}{c}\begin{array}{c}\text { Clean } \\
\text { reads }\end{array} \\
38,089\end{array}$} & \multirow{2}{*}{$\begin{array}{c}\text { Proportion } \\
\text { (\%) }\end{array}$} & \multirow{2}{*}{$\begin{array}{c}\text { AvgLen } \\
413\end{array}$} & \multirow{2}{*}{$\begin{array}{l}\text { Q20 } \\
98.63\end{array}$} & \multirow{2}{*}{$\begin{array}{l}\text { Q30 } \\
95.17\end{array}$} & \multirow{2}{*}{$\begin{array}{c}\text { Total } \\
\text { reads }\end{array}$} & \multirow{2}{*}{$\begin{array}{l}\text { Clean } \\
\text { reads }\end{array}$} & \multirow{2}{*}{$\begin{array}{c}\begin{array}{c}\text { Proportion } \\
\text { (\%) }\end{array} \\
98.55\end{array}$} & \multirow{2}{*}{$\begin{array}{c}\text { AvgLen } \\
226\end{array}$} & \multirow{2}{*}{$\begin{array}{l}\text { Q20 } \\
99.83\end{array}$} & \multirow{2}{*}{$\begin{array}{l}\text { Q30 } \\
99.37\end{array}$} \\
\hline \multirow{5}{*}{ Site 1} & R-1 & & & & & & & & & & & & \\
\hline & $\mathrm{R}-2$ & 41,143 & 34.371 & 83.54 & 413 & 98.66 & 95.28 & 40,872 & 38,929 & 95.24 & 225 & 99.82 & 99.30 \\
\hline & $R-3$ & 43,319 & 37,691 & 87.01 & 413 & 98.62 & 95.10 & 40,753 & 39,207 & 96.21 & 232 & 99.81 & 99.28 \\
\hline & S-1 & 44,224 & 39,050 & 88.30 & 419 & 98.52 & 94.76 & 43,791 & 42,005 & 95.92 & 249 & 99.54 & 98.44 \\
\hline & S-3 & 41,748 & 37,364 & 89.50 & 419 & 98.54 & 94.82 & 40,967 & 35,942 & 87.73 & 262 & 99.55 & 98.45 \\
\hline \multirow{4}{*}{ Site 2} & R-4 & 34,905 & 30,760 & 88.12 & 417 & 98.45 & 94.54 & 44,226 & 39,959 & 90.35 & 252 & 99.75 & 99.10 \\
\hline & $\mathrm{R}-5$ & 41,833 & 36,680 & 87.68 & 416 & 98.55 & 94.93 & 42,358 & 40,274 & 95.08 & 246 & 99.72 & 99.00 \\
\hline & $\mathrm{R}-6$ & 43,612 & 38,426 & 88.11 & 414 & 98.47 & 94.70 & 43,686 & 42,656 & 97.64 & 253 & 99.68 & 98.86 \\
\hline & S-4 & 44,197 & 39,884 & 90.24 & 417 & 98.50 & 94.65 & 42,357 & 38,430 & 90.72 & 266 & 99.54 & 98.43 \\
\hline \multirow{5}{*}{ Site 3} & $\mathrm{R}-8$ & 40,593 & 34,748 & 85.60 & 415 & 98.55 & 94.89 & 41,706 & 39,722 & 95.24 & 247 & 99.76 & 99.13 \\
\hline & $R-9$ & 41,556 & 34,434 & 82.86 & 415 & 98.46 & 94.62 & 40,768 & 37,212 & 91.28 & 238 & 99.83 & 99.35 \\
\hline & S-7 & 42,328 & 37,973 & 89.71 & 419 & 98.54 & 94.80 & 41,338 & 41,156 & 99.56 & 258 & 99.65 & 98.80 \\
\hline & S-8 & 42,939 & 38,061 & 88.64 & 418 & 98.47 & 94.58 & 36,779 & 35,699 & 97.06 & 261 & 99.48 & 98.30 \\
\hline & S-9 & 43,066 & 38,837 & 90.18 & 417 & 98.48 & 94.66 & 44,097 & 42,364 & 96.07 & 261 & 99.67 & 98.86 \\
\hline
\end{tabular}


Table 1. The raw sequencing data and quality control of $16 \mathrm{~S}$ and ITS. R-1 to R-9 are samples collected from the rhizosphere. S-1 to S-9 are samples collected from the bulk soil. AvgLen = average lengths of the clean reads. Q20 and Q30 are the ratio of bases with quality value more than 20 and 30 (error rates of less than $1 \%$ and $0.1 \%$, respectively) in the clean reads. 


\section{Table 2 (on next page)}

OTU clustering of sequence data.

R-1 to R-9 are samples collected from the rhizosphere. S-1 to S-9 are samples collected from the bulk soil. Taxon reads: annotated clean reads that are used to set up OTUs. Unclassified reads: reads without annotations. Singletons: single reads that could not be clustered to any OTUs (These reads were not subjected to further analysis). 


\begin{tabular}{|c|c|c|c|c|c|c|c|c|c|c|c|}
\hline \multirow{2}{*}{\multicolumn{2}{|c|}{$\begin{array}{l}18 \text { samples } \\
\text { from } 3 \text { sites }\end{array}$}} & \multicolumn{5}{|c|}{$16 S$} & \multicolumn{5}{|c|}{ ITS } \\
\hline & & Clean reads & Taxon reads & Unclassified & Singletons & OTUs & Clean reads & Taxon reads & Unclassified & Singletons & OTUs \\
\hline \multirow{6}{*}{ Site 1} & R-1 & 38,089 & 30,857 & 204 & 7,028 & 2,332 & 43,394 & 42,353 & 0 & 1,041 & 452 \\
\hline & $\mathrm{R}-2$ & 34,371 & 25,898 & 150 & 8,323 & 2,216 & 38,815 & 38,013 & 0 & 802 & 340 \\
\hline & $R-3$ & 37,691 & 30,274 & 216 & 7,201 & 2,433 & 39,207 & 38,570 & 0 & 637 & 327 \\
\hline & S-1 & 39,050 & 25,265 & 789 & 12,996 & 3,001 & 42,005 & 40,290 & 0 & 1,715 & 734 \\
\hline & $\mathrm{S}-2$ & 34,300 & 22,183 & 768 & 11,349 & 2,684 & 40,097 & 38,667 & 0 & 1,430 & 531 \\
\hline & $\mathrm{S}-3$ & 37,364 & 29,709 & 930 & 6,725 & 3,241 & 35,942 & 34,819 & 0 & 1,123 & 564 \\
\hline \multirow{6}{*}{ Site 2} & R-4 & 30,760 & 22,398 & 357 & 8,005 & 2,799 & 39,959 & 38,325 & 0 & 1,634 & 474 \\
\hline & R-5 & 36,680 & 25,620 & 434 & 10,626 & 2,999 & 40,274 & 38,028 & 0 & 2,246 & 552 \\
\hline & $\mathrm{R}-6$ & 38,426 & 28,191 & 429 & 9,806 & 3,000 & 42,656 & 41,632 & 0 & 1,024 & 532 \\
\hline & S-4 & 39,884 & 30,053 & 1,206 & 8,625 & 3,190 & 38,430 & 36,290 & 0 & 2,140 & 340 \\
\hline & $S-5$ & 37,796 & 26,394 & 993 & 10,409 & 3,018 & 36,392 & 34,976 & 0 & 1,416 & 426 \\
\hline & S-6 & 36,649 & 25,629 & 1,344 & 9,676 & 2,966 & 41,628 & 40,503 & 0 & 1,125 & 462 \\
\hline \multirow{6}{*}{ Site 3} & $\mathrm{R}-7$ & 33,739 & 24,797 & 243 & 8,699 & 2,695 & 42,305 & 41,409 & 0 & 896 & 556 \\
\hline & $\mathrm{R}-8$ & 34,748 & 25,363 & 323 & 9,062 & 2,795 & 39,722 & 38,696 & 0 & 1,026 & 466 \\
\hline & R-9 & 34,434 & 24,903 & 199 & 9,332 & 2,612 & 37,212 & 36,505 & 0 & 707 & 412 \\
\hline & S-7 & 37,973 & 30,256 & 1,777 & 5,940 & 2,837 & 41,156 & 40,657 & 0 & 499 & 244 \\
\hline & $\mathrm{S}-8$ & 38,061 & 27,511 & 1,366 & 9,184 & 3,014 & 35,699 & 35,165 & 0 & 534 & 144 \\
\hline & S-9 & 38,837 & 27,550 & 1,795 & 9,492 & 2,987 & 42,364 & 41,909 & 0 & 455 & 199 \\
\hline
\end{tabular}


Table 2. OTU clustering of sequence data. R-1 to R-9 are samples collected from the rhizosphere. S-1 to S-9 are samples collected from the bulk soil. Taxon reads: annotated clean reads that are used to set up OTUs. Unclassified reads: reads without annotations.

Singletons: single reads that could not be clustered to any OTUs (These reads were not subjected to further analysis). 